\title{
Viral apoptotic mimicry: an immune evasion strategy developed by the hepatitis $B$ virus?
}

\section{Peter Vanlandschoot and Geert Leroux-Roels}

Center for Vaccinology, Dept of Clinical Biology, Microbiology and Immunology, Ghent University Hospital, De Pintelaan 185, 9000 Ghent, Belgium.

The co-existence of viruses and organisms for millions of years has influenced the evolution of both. Various viral strategies to enter a host and take over the control of cells to produce virus progeny have developed. Several antiviral (immune) responses have also been developed. The apoptotic death program is a conserved feature of eukaryotic cells. In multicellular organisms the binding and engulfment of apoptotic material is considered to be the end stage of the apoptotic process. Because of its importance, it seems probable that viruses have targeted this ancient removal system to suppress immune responses and to establish or maintain infection. The possibility that the hepatitis $B$ virus has evolved such a mechanism, termed 'viral apoptoticlike mimicry', is presented here.

In the past 15 years there has been a tremendous increase in the understanding of the molecular biology of programmed cell death, especially apoptotic cell death. This process is essential for development, morphogenesis and tissue modelling but also has a key role in immune homeostasis [1]. However, without an equally well organized removal process, apoptosis loses its purpose. Indeed, evidence is accumulating that the persistence of apoptotic cells (APCEs) leads to (or contributes to) inflammatory and autoimmune diseases, such as systemic lupus erythematosus. Viruses have shaped the evolution of organisms and vice versa. It is therefore not a surprise that when new cellular and immune mechanisms are discovered, viruses are found that have evolved to manipulate these pathways to their own advantage [2,3]. The APCE removal system is highly conserved, highly effective and designed to prevent tissue damage, inflammation and the development of (auto)immunity. Therefore, it is remarkable that no virus that takes advantage of the machinery to remove APCEs has yet been described.

The hepatitis $\mathrm{B}$ virus (HBV) is one of the most successful human pathogens. Two billion of the six billion people alive today show evidence of past or current infection with this virus and $\sim 350-400$ million people are persistently infected with HBV. Besides infectious virions (Dane particles), HBV-infected hepatocytes produce non-infectious subviral particles [termed hepatitis B

Corresponding author: Peter Vanlandschoot (Peter.Vanlandschoot@rug.ac.be). surface antigen (HBsAg)]. HBsAg consists mainly of spherical particles that can accumulate at $100 \mu \mathrm{g} \mathrm{ml}^{-1}$ in the blood of HBV-infected patients and exceed infectious virions in number by a factor of 100-10 000. HBsAg particles contain viral-encoded membrane proteins $(\mathrm{S}, \mathrm{M}$ and L) and $\sim 30 \%$ (by weight) of host-cell-derived lipids. A possible role for $\mathrm{HBsAg}$ in the establishment and maintenance of HBV persistence has been suggested [4]. It is thought that HBsAg maintains viral persistence by absorbing the virus-neutralizing antibodies. It is further hypothesized that HBsAg-derived peptides saturate MHC molecules and prevent presentation of other viral-derived peptides. HBsAg might also deplete CD4 and CD8 cells by clonal exhaustion and cause cytotoxic T-lymphocyte (CTL)-mediated depletion of T and B cells, which take up $\mathrm{HBsAg}$ into the MHC class I processing pathway. However, no experimental evidence to support these hypotheses has been obtained [4]. Therefore, the reason for the existence, or the possible advantage, of the production of $\mathrm{HBsAg}$ remains elusive. It is, however, remarkable that during acute and chronic infections a clear HBsAg-specific Th-cell and humoral response is difficult to demonstrate, despite the circulation of $\mathrm{HBsAg}$ [4]. Based on recent insights into the mechanism of APCE removal, a hypothesis is presented, in which HBsAg acts like an apoptotic-cell mimic and interacts with the ancient innate APCE removal system to prevent (adaptive) immunity.

HBsAg has anti-inflammatory potential similar to APCEs $\mathrm{HBV}$ is a non-cytopathogenic virus, which might explain why HBV infections often remain unnoticed. However, several studies have suggested a general modulation of antigen-presenting cells [APCs; monocytes, macrophages and dendritic cells (DCs)] in chronically infected patients. An altered balance of Th1 and Th2 responses [5] and defects in the antigen-presenting activity of DCs have been observed [6]. In comparison to peripheral-blood mononuclear cells (PBMCs) from healthy subjects, PBMCs from patients with chronic HBV proliferate less frequently and less vigorously to a non-HBV recall antigen and produce less interferon- $\gamma$ (IFN- $\gamma$ ) and interleukin-12 (IL-12) following stimulation with mitogen [5]. In vitro studies demonstrate a reduced capacity of PBMCs from chronic HBV patients to produce cytokines on stimulation with lipopolysaccharide (LPS). Interestingly, purified HBsAg, 
when added to human monocyte-derived macrophages, efficiently suppresses the production of LPS-induced tumour necrosis factor- $\alpha(\mathrm{TNF}-\alpha)$, vesicular stomatitis virus-induced IFN- $\alpha$ and TNF- $\alpha$-induced granulocytemacrophage-colony stimulating factor (GM-CSF). An increased nuclear degradation of mRNA was demonstrated [7]. Recently, yeast-expressed HBsAg (rHBsAg) was shown to behave as an apoptotic-like particle: $\mathrm{rHBsAg}$ suppressed LPS-induced secretion of proinflammatory cytokines but increased secretion of IL-10 by monocytes $[8,9]$. Similarly, binding and engulfment of APCEs suppresses secretion of proinflammatory cytokines and certain chemokines by macrophages $[10,11]$. This effect of APCEs is also observed when macrophages are stimulated with LPS. Because addition of anti-transforming growth factor- $\beta$ (TGF- $\beta$ ), platelet-activating factor (PAF) receptor anatagonist or indomethacin to APCE- and LPS-treated macrophages restores inflammatory cytokine production, TGF- $\beta$, PAF and prostaglandin $\mathrm{E}_{2}$ are suggested to mediate the decrease of the inflammatory cytokines [10]. Suppression is transcriptionally and translationally regulated, depending on the cytokine or chemokine examined [11]. In contrast to macrophages, monocytes do not engulf APCEs. Nevertheless, the binding of APCEs to monocytes reduces the LPS-induced secretion of TNF- $\alpha$ and IL-1 $\beta$, although increased levels of IL-10 and TGF- $\beta$ can be observed $[12,13]$. Although IL-10 and TGF- $\beta$ are major inhibitors of TNF- $\alpha$ and IL-1 $\beta$ production, these cytokines are not responsible for the reduction in TNF- $\alpha$ and IL-1 $\beta$ [13]. Taken together, these observations suggest an anti-inflammatory potential of $\mathrm{HBsAg}$ similar to APCEs on monocytes and macrophages.

\section{HBsAg interacts with molecules that bind APCEs}

Several cell membrane-bound receptors have been identified that recognize APCEs: the asialoglycoprotein receptor, class A and B scavenger receptors (SR-A and SR-BI), CD36, macrosialin or oxidized low-density lipoprotein (oxLDL) receptor (CD68), lectin-like oxLDL receptor-1 (LOX-1), ATP-binding cassette transporter 1 (ABC1), $\alpha \mathrm{v} \beta 3$ and $\alpha \mathrm{v} \beta 5$ vitronectin receptor, MER tyrosine kinase receptor [a member of the TAM (Tyro-Axl-Mer tyrosine kinase) receptor family, expressed on monocytes and tissues of epithelial and reproductive origin], C1q receptor (calreticulin) adhered to CD91 ( $\alpha 2$ macroglobulin receptor, heat-shock protein receptor or LDL receptor-related protein), complement receptors 3 and 4 (CR3 and CR4), the phosphatidylserine (PtdSer) receptor and CD14. Besides these membrane receptors, several soluble factors have been identified that bind to APCEs: thrombospondin (TSP), C1q, C3 and C4, $\beta 2$-glycoprotein I ( $\beta 2$-GPI), pentraxins [PTX3, C-reactive protein (CRP) and serum amyloid $\mathrm{P}$ (SAP)], growth arrest specific gene- 6 product (gas-6), mannose-binding lectin (MBL), surfactant protein-A and -D (SP-A and SP-D), histidine rich glycoprotein (HRG), vitamin K-dependent protein $\mathrm{S}$ and anti-oxidized phosphatidylcholine (anti-oxPtdCho) antibodies [14-18]. APCEs can display several molecules important for recognition and engulfment. The most important determinant for recognition of APCEs is thought to be PtdSer. 32-GPI, gas-6, SR-A, SR-BI, CD36, CD68, ABC1, LOX-1,
CD14 and the PtdSer receptor all bind PtdSer. Several of these also recognize other charged phospholipids (PLs), such as phosphatidylinositol (PtdIns). Recent observations suggest that oxPtdChos are important recognition molecules displayed by APCEs and oxidation of PtdSer enhances engulfment of APCEs [19,20]. OxPtdCho is a ligand for CRP, scavenger receptors and anti-oxPtdCho antibodies. Interestingly, binding of SAP to APCEs occurs through interaction with phosphatidylethanolamine (PtdEtn) [21] (Fig. 1). HBsAg contains 30\% (by weight) of host-derived lipids. The main lipid components of HBsAg are PLs $(\sim 67 \%)$, cholesteryl ester $(\sim 15 \%)$, cholesterol $(\sim 14 \%)$ and triglycerides $(\sim 3 \%)$. PtdCho accounts for $\sim 90 \%$ of the PLs and PtdEtn accounts for 2-4\%. Trace amounts of PtdSer, sphingomyelin, lysoPtdCho and lysoPtdEtn are present [22]. Because of this PL content, HBsAg might interact with membrane-bound receptors or soluble molecules known to bind PL that are also displayed by APCEs. $\beta 2-$ GPI is a plasma protein with high affinity for charged PLs and a key antigen in the antiphospholipidsyndrome [23]. Attachment of $\beta 2$-GPI to APCEs [24] depends on the presence of charged PL, most probably PtdSer [25]. Only after binding of $\beta 2$-GPI to APCEs, a $\beta 2$-GPI-dependent interaction of the complex with macrophages becomes possible [26]. Binding of $\beta 2-G P I$ to an HBsAg-containing fraction enriched for Dane particles, was reported [27,28]. Recently, it was reported that $\beta 2$-GPI binds preferentially to Dane particles. Experiments using recombinant $\mathrm{HBsAg}$ suggested that this interaction requires binding to myristilated $\mathrm{L}$ protein and depends on charged PLs, such as PtdSer. Moreover, oxidation of the PLs is suggested to enhance binding [29]. Nevertheless, yeast and insect cell-expressed HBsAg, as well as plasma HBsAg, have been demonstrated to bind $\beta 2-G P I$. Because of the enormous amounts of $\mathrm{HBsAg}$, the lower interaction avidity with $\beta 2$-GPI might still have a substantial anti-inflammatory effect. Another molecule demonstrated to bind APCEs and to bind several PLs is CD14 [30]. A soluble form of CD14 (sCD14) is present in circulation. In chronic hepatitis B infections, levels of sCD14 are increased but, interestingly, an inverse correlation between HBsAg levels and sCD14 levels is found (S. Steyaert et al., unpublished). Recently rHBsAg was shown to behave as an apoptotic-like particle [8,9]. Additionally, rHBsAg binds to monocytes through interaction with the LPS-binding protein and CD14 [31]. Remarkably, plasma HBsAg does not have these characteristics and the low PtdSer content (1-2\%) and the lack of PtdIns in HBsAg could cause this difference. Although PtdIns is abundant (10-20\%) in the endoplasmic reticulum (ER) membrane where HBsAg particles assemble, it is surprisingly not present in $\mathrm{HBsAg}$. However, HBsAg, when expressed in mammalian cells [such as Chinese hamster ovary $(\mathrm{CHO})$, human hepatoma cells and mouse fibroblast cells] or yeast cells (Saccharomyces cerevisae and Hansenula polymorpha) always contains $\geq 4-7 \%$ of PtdSer and/or PtdIns. Considering that different PL-transfer molecules are present in the circulation, the question can be raised of whether or not the PL content of $\mathrm{HBsAg}$ in circulation is dynamic and changes over time (or even during storage of plasma). Soon after the discovery of 

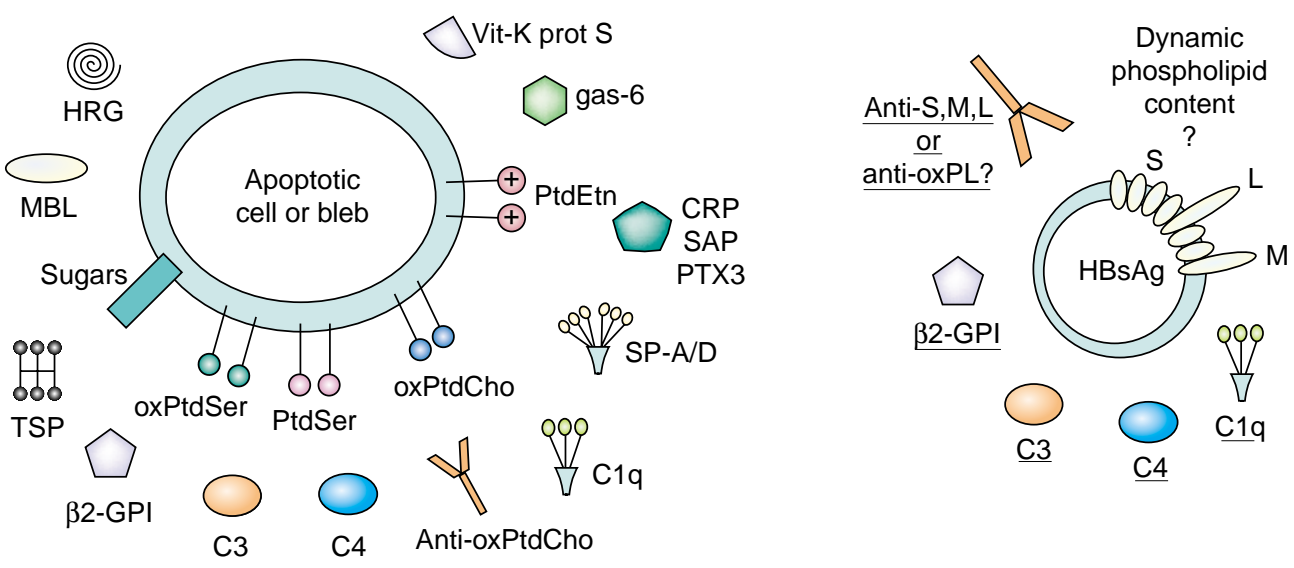

C3 $\quad$ C4 Anti-oxPtdCho

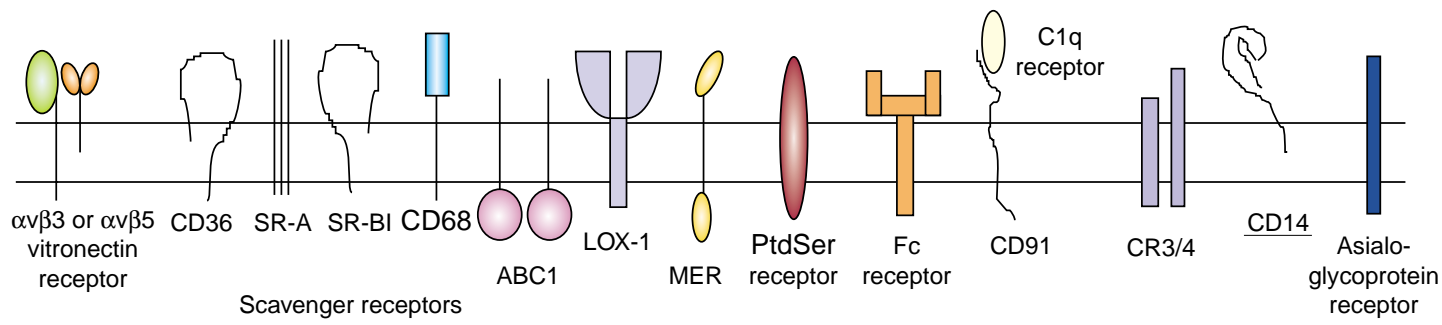

$\overline{\text { TRENDS in Immunology }}$

Fig. 1. Soluble factors and cellular receptors involved in binding and phagocytosis of apoptotic cells. Those molecules that have been shown to bind to, or circulate in, complexes with hepatitis $B$ surface antigen (HBsAg) are depicted separately and are underlined. Abbreviations: ABC1, ATP-binding cassette transporter 1 ; anti- $\mathrm{L}$, anti- $\mathrm{L}$ antibody; anti-M, anti-M antibody; anti-S, anti-S antibody; anti-oxPL, anti-oxidized phospholipid antibody; anti-oxPtdCho, anti-oxidized phosphatidylcholine antibody; $\beta 2-G P I$, B2-glycoprotein I; C1q, complement component 1q; C3, complement component 3; C4, complement component 4; CD14, lipopolysaccharide receptor; CD36, platelet adhesion molecule; CD68, macrosialin or oxidized low-density lipoprotein (oxLDL) receptor; CD91, $\alpha 2$ macroglobulin receptor, heat shock protein receptor or LDL receptorrelated protein; CR3/4, complement receptor 3/4; CRP, C-reactive protein; gas-6, growth arrest specific gene-6 product; HRG, histine rich glycoprotein; LOX-1, lectin-like oxidized LDL receptor-1; MBL, mannose-binding lectin; MER, MER tyrosine kinase receptor; oxPtdCho, oxidized phosphatidylcholine; oxPtdSer, oxidized phosphatidylserine; PtdEtn, phosphatidylethanolamine; PTX3, pentraxin 3; SAP, serum amyloid P; SP-A/D, surfactant protein A/D; SR-A, class A scavenger receptor; SR-BI, BI scavenger receptor; TSP, thrombospondin; Vit-K prot S, vitamin-K-dependent protein S.

HBsAg in circulation, the presence of HBsAg in immune complexes containing different amounts and combinations of IgM and/or IgG was noticed. It is often assumed that the antibodies found in the HBsAg-immune complexes are directed against the viral proteins of $\mathrm{HBsAg}$. However, no evidence for this has been presented. Taking into account the high PL content of the particles, and as oxidation of these PLs might occur during circulation, the possibility is raised here that PL-specific or anti-oxPL antibodies are present in the HBsAg immune complexes. Such antibodies develop early in life, selected by the presence of oxidized phospholipids [19]. It is thought that APCEs opsonized by anti-oxPtdCho antibodies are removed by Fc-receptor bearing cells. If oxidation of the PLs of HBsAg occurs during circulation, also other molecules, such as CRP and scavenger receptors, might perhaps interact with HBsAg. Complement factors are also known to bind to unique PL displayed by APCEs [32]. The circulating HBsAg (immune) complexes can contain several complement molecules: C1q, C1r, C1s, C4 and C3. More than 20 years ago, evidence for binding of $\mathrm{C} 1 \mathrm{q}$ to $\mathrm{HBsAg}$, possibly in a complex with polymerized human albumin, was presented [33]. C1q is a key molecule in the removal of APCEs because the lack of C1q (but also C4) is associated with decreased engulfment of APCEs and sometimes development of disease in mice [15]. A complex interaction between APCEs, C1q or SP-A or SP-D and calreticulin,
MBL and CD91 has been proposed [34,35]. Additionally, the anti-inflammatory interaction of CRP with APCEs requires C1q [36] and the circulation of apoptotic blebs in association with $\mathrm{C} 1 \mathrm{q}$ was demonstrated [37]. Taken together, binding of $\mathrm{HBsAg}$ to (or $\mathrm{HBsAg}$ complexed with) APCE-binding molecules has been demonstrated. Depending on a (dynamic) PL content and/or oxidation of $\mathrm{PLs}$, interactions with other molecules is considered possible.

\section{Are circulating complement-HBsAg complexes preventing adaptive immunity towards the viral envelope proteins?}

A recent paper described the unexpected importance of complement and complement receptors (CR3 and CR4) in the uptake of circulating APCEs by splenic marginal zone DCs. Following the phagocytosis of complement-opsonized APCEs, decreased levels of proinflammatory cytokine secretion, without an effect on TGF- $\beta$ secretion, was observed. This cytokine switch is thought to diminish antigen-driven T-cell stimulation by DCs that ingested APCEs and this might lead to immune unresponsiveness (peripheral tolerance) [38,39]. Based on these observations it is proposed that circulating $\mathrm{HBsAg}$, complexed with complement factors (C1q, C3 and C4) might similarly be taken up by DCs. This might lead to induction of tolerance towards the viral envelope proteins. This mechanism could 
explain why an adequate anti-envelope immune response is lacking despite the presence of HBsAg and why only after elimination of infection, antibodies become detectable.

\section{Concluding remarks}

The evidence for the hypothesis of 'viral apoptotic mimicry' gathered here mainly comes from (older) studies that did not focus on viral evasion of immunity by the HBV. It is now hoped that this hypothesis results in renewed interest on the possible role of HBsAg in immune evasion. Of major importance is to determine the PL content of HBsAg, when freshly secreted by hepatocytes, and to determine if this content changes during circulation. If changes in PL content or oxidation of PLs occur, the use of $\mathrm{HBsAg}$ reconstituted with specific PLs might lead to the identification of serum proteins and cellular receptors known to bind APCEs. Obviously the major APC targeted by HBsAg needs to be identified. Finally, although future research might reject this hypothesis for HBV, it is considered almost inevitable that sooner or later viruses will be identified that have targeted the APCE removal system to avoid (adaptive) immunity.

\section{References}

1 Fiers, W. et al. (1999) More than one way to die: apoptosis, necrosis and reactive oxygen damage. Oncogene 18, 7719-7730

2 Tortorella, D. et al. (2000) Viral subversion of the immune system. Annu. Rev. Immunol. 18, 861-926

3 Alcami, A. and Koszinowski, U.H. (2000) Viral mechanisms of immune evasion. Trends Microbiol. 3, 379-386

4 Milich, D.R. (1997) Immune response to the hepatitis B virus: infection, animal models, vaccination. Viral Hepatitis. Rev. 3, 63-103

5 Livingston, B.D. et al. (1999) Altered helper T-lymphocyte function associated with chronic hepatitis B virus infection and its role in response to therapeutic vaccination in humans. J. Immunol. 162, 3088-3095

6 Akbar, S.M. et al. (2001) Dendritic cells and chronic hepatitis virus carriers. Intervirology 44, 199-208

7 Jochum, C. et al. (1990) Immunosuppressive function of hepatitis B antigens in vitro: role of endoribonuclease $\mathrm{V}$ as one potential trans inactivator for cytokines in macrophages and human hepatoma cells. J. Virol. 64, 1956-1963

8 Vanlandschoot, P. et al. (2002) Hepatitis B surface antigen suppresses the activation of monocytes through interaction with a serum protein and a monocyte-specific receptor. J. Gen. Virol. 83, 1281-1289

9 Vanlandschoot, P. et al. (2002) Recombinant HBsAg, an apoptotic-like lipoprotein, interferes with the LPS-induced phosphorylation of ERK-1/2 and JNK-1/2 in monocytes. Biochem. Biophys. Res. Commun. 297, 486-491

10 Fadok, V.A. et al. (1998) Macrophages that have ingested apoptotic cells in vitro inhibit proinflammatory cytokine production through autocrine/paracrine mechanisms involving TGF- $\beta, \mathrm{PGE}_{2}$ and PAF. J. Clin. Invest. 101, 890-898

11 McDonald, P.P. et al. (1999) Transcriptional and translational regulation of inflammatory mediator production by endogenous TGF- $\beta$ in macrophages that have ingested apoptotic cells. J. Immunol. 163, 6164-6172

12 Voll, R.E. et al. (1997) Immunosuppressive effects of apoptotic cells. Nature 390, 350-351

13 Byrne, A. and Reen, D.J. (2002) Lipopolysaccharide induces rapid production of IL-10 by monocytes in the presence of apoptotic neutrophils. J. Immunol. 168, 1968-1977

14 Fadok, V.A. et al. (2001) Phagocyte receptors for apoptotic cells: recognition, uptake and consequences. J. Clin. Invest. 108, 957-962

15 Manfredi, A.A. et al. (2002) The disposal of dying cells in living tissues. Apoptosis 7, 153-161
16 Franc, N.C. (2002) Phagocytosis of apoptotic cells in mammals Caenorhabditis elegans and Drosophila melanogaster: molecular mechanisms and physiological consequences. Front. Biosci. 7, $1298-1313$

17 Gorgani, N.N. et al. (2002) Histidine-rich glycoprotein binds to DNA and Fc $\gamma R I$ and potentiates the ingestion of apoptotic cells by macrophages. J. Immunol. 169, 4745-4751

18 Webb, J.H. et al. (2002) Vitamin K-dependent protein S localizing complement regulator $\mathrm{C} 4 \mathrm{~b}$-binding protein to the surface of apoptotic cells. J. Immunol. 169, 2580-2586

19 Hazen, S.L. and Chisolm, G.M. (2002) Oxidized phosphatidylcholines: pattern recognition ligands for multiple pathways of the innate immune response. Proc. Natl. Acad. Sci. U. S. A. 99, 12515-12517

20 Kagan, V.A. et al. (2002) A role for oxidative stress in apoptosis: oxidation and externalization of phosphatidylserine is required for macrophage clearance of cells undergoing Fas-mediated apoptosis. J. Immunol. 169, 487-499

21 Familian, A. et al. (2001) Chromatin-independent binding of serum amyloid P component to apoptotic cells. J. Immunol. 167, 647-654

22 Gavilanes, F. et al. (1982) Structure of hepatitis B surface antigen. Characterization of the lipid components and their association with the viral proteins. J. Biol. Chem. 257, 7770-7777

23 Lozier, J. et al. (1984) Complete amino-acid sequence of human ß2-glycoprotein. Proc. Natl. Acad. Sci. U. S. A. 81, 3640-3644

24 Price, B.E. et al. (1996) Anti-phospholipid autoantibodies bind to apoptotic but not viable thymocytes in a $\beta 2$-glycoprotein I-dependent manner. J. Immunol. 157, 2201-2208

25 Balasubramanian, K. et al. (1997) Immune clearance of phophatidylsreine-expressing cells by phagocytes. The role of $\beta 2$-glycoprotein I in macrophage recognition. J. Biol. Chem. 272, 31113-31117

26 Balasubramanian, K. and Schroit, A.J. (1998) Characterization of phosphatidylserine-dependent $\beta 2$-glycoprotein I macrophage interactions. Implications for apoptotic-cell clearance by phagocytes. J. Biol. Chem. 273, 29272-29277

27 Mehdi, M. et al. (1994) Hepatitis B virus surface antigen binds to apolipoprotein H. J. Virol. 68, 2415-2424

28 Mehdi, M. et al. (1996) An altered form of apolipoprotein H binds hepatitis B surface antigen most efficiently. Virology 217, 58-66

29 Stefas, I. et al. (2001) Hepatitis B virus Dane particles bind to human plasma apolipoprotein H. Hepatology 33, 207-217

30 Gregory, C.D. (2000) CD14-dependent clearance of apoptotic cells: relevance to the immune system. Curr. Opin. Immunol. 12, 27-34

31 Vanlandschoot, P. et al. (2002) LPS binding protein and CD14-dependent attachment of hepatitis B surface antigen to monocytes is determined by the phospholipid moiety of the particles. J. Gen. Virol. 83, $2279-2289$

32 Mevorach, D. et al. (1998) Complement-dependent clearance of apoptotic cells by human macrophages. J. Exp. Med. 188, 2313-2320

33 Milich, D.R. et al. (1981) Interactions between polymerized human albumin, hepatitis B surface antigen and complement: II. Involvement of $\mathrm{C} 1 \mathrm{q}$ in or near the hepatitis $\mathrm{B}$ surface antigen receptor for polyalbumin. J. Med. Virol. 7, 193-204

34 Ogden, C.A. et al. (2001) C1q and mannose binding lectin engagement of cell-surface calreticulin and CD91 initiates macropinocytosis and uptake of apoptotic cells. J. Exp. Med. 194, 781-795

35 Vandivier, R.W. et al. (2002) Role of surfactant proteins A, D and C1q in the clearance of apoptotic cells in vivo and in vitro: calreticulin and CD91 as a common collectin receptor complex. J. Immunol. 169 3978-3986

36 Gershov, D. et al. (2000) C-reactive protein binds to apoptotic cells, protects the cells from assembly of the terminal complement components and sustains an anti-inflammatory innate immune response: implications for systemic autoimmunity. J. Exp. Med. 192, 1353-1364

37 Nauta, A.J. et al. (2002) Direct binding of C1q to apoptotic cells and cell blebs induces complement activation. Eur. J. Immunol. 32, 1726-1736

38 Steinman, R.M. et al. (2000) The induction of tolerance by dendritic cells that have captured apoptotic cells. J. Exp. Med. 191, 411-416

39 Morelli, A.E. et al. (2003) Internalization of circulating apoptotic cells by splenic marginal-zone dendritic cells: dependence on complement receptors and effect on cytokine production. Blood 101, 611-620 